DSF-09/2002

\title{
SEESAW MECHANISM, BARYON ASYMMETRY AND NEUTRINOLESS DOUBLE BETA DECAY
}

\author{
D. Falcone \\ Dipartimento di Scienze Fisiche, Università di Napoli, \\ Complesso di Monte S. Angelo, Via Cintia, Napoli, Italy
}

\begin{abstract}
A simplified but very instructive analysis of the seesaw mechanism is here performed. Assuming a nearly diagonal Dirac neutrino mass matrix, we study the forms of the Majorana mass matrix of right-handed neutrinos, which reproduce the effective mass matrix of left-handed neutrinos. As a further step, the important effect of a non diagonal Dirac neutrino mass matrix is explored. The corresponding implications for the baryogenesis via leptogenesis and for the neutrinoless double beta decay are reviewed. We propose two distinct models where the baryon asymmetry is enhanced.
\end{abstract}




\section{INTRODUCTION}

The seesaw mechanism [1] is a simple framework to account for the small effective mass of the left-handed neutrino. It requires only a modest extension of the minimal standard model, namely the addition of the right-handed neutrino. As a consequence of this inclusion, both a Dirac mass term for the neutrino and a Majorana mass term for the right-handed neutrino are allowed. While the Dirac mass, $m_{\nu}$, is expected to be of the same order of magnitude as the quark or charged lepton mass, the Majorana mass of the right-handed neutrino, $m_{R}$, is not constrained and may be very large. If this is the case, a small effective Majorana mass for the left-handed neutrino, $m_{L} \simeq\left(m_{\nu} / m_{R}\right) m_{\nu}$, is generated.

At the same time, the out-of-equilibrium decay of the heavy neutrino can produce a baryon asymmetry through the so-called baryogenesis via leptogenesis mechanism [2, 3]. Hence, the existence of the heavy Majorana neutrino may explain both the smallness of the effective neutrino mass and the baryon asymmetry in the universe. Moreover, the Majorana nature of the light neutrino, generated by the Majorana nature of the heavy neutrino, allows the neutrinoless double beta decay, because of lepton number violation at high energy [4. Thus the mass scale of the heavy neutrino could be a new fundamental scale in physics.

For three generations of fermions, the light neutrino mass matrix $M_{L}$ as well as the baryon asymmetry $Y_{B}$ depend on the Dirac neutrino mass matrix $M_{\nu}$ and the heavy neutrino mass matrix $M_{R}$. In this paper we describe, in a simplified but instructive approach, the structure of $M_{\nu}$ and $M_{R}$ within the seesaw mechanism and the consequences for the baryon asymmetry generated in the baryogenesis via leptogenesis mechanism and for the neutrinoless double beta decay.

As a first approximation, we assume a diagonal form for the Dirac neutrino mass matrix, and then the effect of a non diagonal form is analyzed. The prediction for the neutrinoless double beta decay can remain unchanged because for a fixed $M_{L}$ and any choice of $M_{\nu}$ one can find a certain $M_{R}$ which reproduces $M_{L}$ through the seesaw formula, while the impact on the amount of baryon asymmetry is significant, because those $M_{R}$ and $M_{\nu}$ determine $Y_{B}$ through the leptogenesis formula.

The outline of the paper is the following. In section II we give an approximate description of the effective neutrino mass matrix. In sections III and IV we briefly discuss the seesaw mechanism and the baryogenesis via leptogenesis, respectively. In section $\mathrm{V}$

the forms of the heavy neutrino mass matrix, and their implications for the amount of baryon asymmetry and the rate for neutrinoless double beta decay are reviewed, according to different mass spectra of light neutrinos. In this section we assume a diagonal Dirac 
neutrino mass matrix. The important effect of a non diagonal Dirac neutrino mass matrix on the amount of baryon asymmetry is explored in section VI, where we also propose two different models of mass matrices, which produce an enhancement of the baryon asymmetry. Finally, in section VII we summarize the subject.

\section{THE EFFECTIVE NEUTRINO MASS MATRIX}

The lepton mixing matrix $U$ (called the Maki-Nakagawa-Sakata (MNS) matrix [5]), which relates mass eigenstates to flavor eigenstates, by means of the unitary transformation $\nu_{\alpha}=U_{\alpha i} \nu_{i}(\alpha=e, \mu, \tau ; i=1,2,3)$, can be parametrized as the standard form of the Cabibbo-Kobayashi-Maskawa (CKM) quark mixing matrix [6] (including a phase $\delta$ ) times a diagonal phase matrix $P=\operatorname{diag}\left(\mathrm{e}^{\mathrm{i} \varphi_{1} / 2}, \mathrm{e}^{\mathrm{i} \varphi_{2} / 2}, 1\right)$. The two phases $\varphi_{1}$ and $\varphi_{2}$ are present only if the effective neutrino is a Majorana particle, and thus they are sometimes called the Majorana phases, in contrast with the phase $\delta$ which is called the Dirac phase. Moreover, contrary to quark mixings, which are small, lepton mixings can be large. In fact, the mixing of atmospheric neutrinos, related to $U_{\mu 3}$, is almost maximal, while the mixing of solar neutrinos, related to $U_{e 2}$, may be large or small, although the large mixing is favoured [7]. In the case of double large mixing, the lepton mixing matrix is given by

$$
U \simeq\left(\begin{array}{ccc}
\frac{1}{\sqrt{2}} & \frac{1}{\sqrt{2}} & \epsilon \\
-\frac{1}{2}(1+\epsilon) & \frac{1}{2}(1-\epsilon) & \frac{1}{\sqrt{2}} \\
\frac{1}{2}(1-\epsilon) & -\frac{1}{2}(1+\epsilon) & \frac{1}{\sqrt{2}}
\end{array}\right)
$$

while for single large mixing it is given by

$$
U \simeq\left(\begin{array}{ccc}
1 & 0 & \epsilon \\
-\frac{\epsilon}{\sqrt{2}} & \frac{1}{\sqrt{2}} & \frac{1}{\sqrt{2}} \\
-\frac{\epsilon}{\sqrt{2}} & -\frac{1}{\sqrt{2}} & \frac{1}{\sqrt{2}}
\end{array}\right)
$$

see for example Ref. [8]. As we said, the double large mixing is favoured. The mixing $\epsilon=U_{e 3}$ is very small, $\epsilon \lesssim 0.1$, according to the result of the Chooz experiment [9]. If we call $D_{L}$ the diagonal matrix of light neutrino masses,

$$
D_{L}=\operatorname{diag}\left(m_{1}, m_{2}, m_{3}\right)
$$

then, in the basis where the charged lepton mass matrix is diagonal, $M_{e}=D_{e}$, we get 


$$
M_{L}=U D_{L} U^{T} .
$$

In the flavor basis we have $M_{L}=U_{e} U D_{L} U^{T} U_{e}^{T}$, where $U_{e}$ diagonalizes $M_{e}$ by $U_{e}^{T} M_{e} U_{e}$, where $M_{e}$ is here supposed to be symmetric. We do not consider general phases in $U$. However, we allow the masses $m_{1}$ and $m_{2}$ to be both positive and negative, corresponding to phases $\varphi_{1,2}=0$ and $\varphi_{1,2}=\pi$, respectively, in the lepton mixing matrix. In such a way, we consider the two extreme cases for $\varphi_{1,2}$ and the general case should be intermediate between them.

Let us call the elements of $M_{L}$ as $M_{\alpha \beta}$ with $\alpha=e, \mu, \tau$ and $\beta=e, \mu, \tau$. Then, for the double large mixing we have the approximate expressions

$$
\begin{gathered}
M_{e e}=\frac{m_{1}}{2}+\frac{m_{2}}{2}+\epsilon^{2} m_{3} \\
M_{e \mu}=-\frac{m_{1}}{2 \sqrt{2}}(1+\epsilon)+\frac{m_{2}}{2 \sqrt{2}}(1-\epsilon)+\frac{\epsilon m_{3}}{\sqrt{2}} \\
M_{e \tau}=\frac{m_{1}}{2 \sqrt{2}}(1-\epsilon)-\frac{m_{2}}{2 \sqrt{2}}(1+\epsilon)+\frac{\epsilon m_{3}}{\sqrt{2}} \\
M_{\mu \mu}=\frac{m_{1}}{4}(1+\epsilon)^{2}+\frac{m_{2}}{4}(1-\epsilon)^{2}+\frac{m_{3}}{2} \\
M_{\mu \tau}=-\frac{m_{1}}{4}\left(1-\epsilon^{2}\right)-\frac{m_{2}}{4}\left(1-\epsilon^{2}\right)+\frac{m_{3}}{2} \\
M_{\tau \tau}=\frac{m_{1}}{4}(1-\epsilon)^{2}+\frac{m_{2}}{4}(1+\epsilon)^{2}+\frac{m_{3}}{2}
\end{gathered}
$$

and for the single large mixing the corresponding expressions

$$
\begin{gathered}
M_{e e}=m_{1}+\epsilon^{2} m_{3} \\
M_{e \mu}=-\frac{\epsilon m_{1}}{\sqrt{2}}+\frac{\epsilon m_{3}}{\sqrt{2}} \\
M_{e \tau}=-\frac{\epsilon m_{1}}{\sqrt{2}}+\frac{\epsilon m_{3}}{\sqrt{2}} \\
M_{\mu \mu}=\frac{\epsilon^{2} m_{1}}{2}+\frac{m_{2}}{2}+\frac{m_{3}}{2} \\
M_{\mu \tau}=\frac{\epsilon^{2} m_{1}}{2}-\frac{m_{2}}{2}+\frac{m_{3}}{2} \\
M_{\tau \tau}=\frac{\epsilon^{2} m_{1}}{2}+\frac{m_{2}}{2}+\frac{m_{3}}{2} .
\end{gathered}
$$

Of course, both $M_{L}$ and $M_{R}$ are symmetric matrices. Note that for the single large mixing we have $M_{e \mu}=M_{e \tau}$ and $M_{\mu \mu}=M_{\tau \tau}$.

The element $M_{e e}=U_{e i}^{2} m_{i}$ is involved in the neutrinoless double beta decay. The experimental upper bound for $\left|M_{e e}\right|$, obtained from non observation of the process, is 
$\left|M_{e e}\right|<0.38 h$ [10], where the factor $h=0.6-2.8$ [11] is present because of the uncertainty in the calculation of the nuclear matrix element. The recent positive evidence [12] is controversial [11.13] and is not used here. The parameter $M_{e e}$ is the unique element in $M_{L}$ which can be tested in a direct way, because for the other elements the theoretical prediction is very much below the experimental data [14].

From the study of oscillations of atmospheric and solar neutrinos we know that $\left|m_{3}^{2}-m_{2}^{2}\right| \gg\left|m_{2}^{2}-m_{1}^{2}\right|$, so that there are three main mass spectra for the light neutrino, the normal hierarchy $m_{3}^{2} \gg m_{2}^{2}, m_{1}^{2}$, the inverse hierarchy $m_{1}^{2} \simeq m_{2}^{2} \gg m_{3}^{2}$, and the nearly degenerate spectrum $m_{1}^{2} \simeq m_{2}^{2} \simeq m_{3}^{2}$ (see Ref. [15]). In particular, from atmospheric oscillations we get $\left|m_{3}^{2}-m_{2}^{2}\right| \sim 10^{-3} \mathrm{eV}^{2}$, while from solar oscillations $\left|m_{2}^{2}-m_{1}^{2}\right| \sim 10^{-5} \mathrm{eV}^{2}$ for the large mixing and $\left|m_{2}^{2}-m_{1}^{2}\right| \sim 10^{-6} \mathrm{eV}^{2}$ for the small mixing. Then, with the normal hierarchy we get $m_{3}^{2} \sim 10^{-3} \mathrm{eV}^{2}$, and with the inverse hierarchy $m_{1,2}^{2} \sim 10^{-3} \mathrm{eV}^{2}$. For the nearly degenerate spectrum we expect $m_{1,2,3}^{2}$ around $1 \mathrm{eV}^{2}$. In fact, the experimental upper bound on the parameter $m_{\nu_{e}}=\left(U_{e i}^{2} m_{i}^{2}\right)^{1 / 2}$, obtained from the endpoint energy of electrons in single beta decay, is $m_{\nu_{e}}<2.5 \mathrm{eV}$ [16]. In contrast with $M_{e e}$, in $m_{\nu_{e}}$ cancellations cannot occur. If for the normal hierarchy also $m_{2}^{2} \gg m_{1}^{2}$ is assumed, then we get $m_{2}^{2} \sim 10^{-5} \mathrm{eV}^{2}$ for the large mixing and $m_{2}^{2} \sim 10^{-6} \mathrm{eV}^{2}$ for the small mixing.

As a matter of fact, for solar neutrinos there are at least three oscillation solutions with a large mixing angle [7]: the large mixing angle (LMA) matter oscillation with $\left|m_{2}^{2}-m_{1}^{2}\right| \sim 10^{-5} \mathrm{eV}^{2}$, the low-mass (LOW) matter oscillation with $\left|m_{2}^{2}-m_{1}^{2}\right| \sim 10^{-7}$ $\mathrm{eV}^{2}$, and the vacuum oscillation (VO) with $\left|m_{2}^{2}-m_{1}^{2}\right| \sim 10^{-10} \mathrm{eV}^{2}$. In our paper we refer mainly to the LMA solution which is the most favoured [7].

Zeroth order forms for $M_{L}$ can be obtained by setting $\epsilon=0$ and $\left(m_{1}, m_{2}, m_{3}\right)$ equal to $(0,0,1),(1,-1,0),(1,1,0),(1,1,1),(-1,1,1),(1,-1,1),(-1,-1,1)$. We call these mass spectra $A, B_{1}, B_{2}, C_{0}, C_{1}, C_{2}, C_{3}$, respectively. Of course, type $A$ is the normal hierarchy, type $B$ the inverse hierarchy, and type $C$ nearly degenerate. In the present paper we write only the zeroth order form of $M_{L}$, although we study the full element $M_{e e}$. For a more detailed description of $M_{L}$ see for example [8].

\section{SEESAW MECHANISM}

Since in the standard model with right-handed neutrinos the Dirac neutrino mass is generated on the same footing as the up quark masses, and the charged lepton masses

on the same footing as the down quark masses, for the corresponding mass matrices we first assume the relations $M_{\nu} \simeq M_{u}$ and $M_{e} \simeq M_{d}$, that is

$$
M_{\nu} \simeq \operatorname{diag}\left(m_{u}, m_{c}, m_{t}\right),
$$


and $M_{e} \simeq \operatorname{diag}\left(m_{e}, m_{\mu}, m_{\tau}\right)$, where we neglect the small Dirac lepton mixing analogous to the quark mixing. In other terms, we set $U_{e} \simeq \mathbb{1}$ and $U_{\nu} \simeq \mathbb{1}$, where $U_{e}$ diagonalizes $M_{e}$ and $U_{\nu}$ diagonalizes $M_{\nu}$. As a matter of fact, the mass hierarchy of charged leptons is similar to that of down quarks. See Ref. [17] for a summary on quark and lepton mass matrices. Because of the seesaw mechanism, we have

$$
M_{L} \simeq M_{\nu} M_{R}^{-1} M_{\nu}
$$

where the heavy neutrino masses $M_{1}, M_{2}, M_{3}$ have to be much larger than the elements of the matrix $M_{\nu}$, which is here supposed to be symmetric. Inverting such a formula, the heavy neutrino mass matrix can be achieved,

$$
M_{R} \simeq M_{\nu} M_{L}^{-1} M_{\nu}
$$

Since $M_{L}^{-1}=U D_{L}^{-1} U^{T}$, we can get $M_{L}^{-1}$ from $M_{L}$ by changing $m_{i}$ with $1 / m_{i}$ in (4). We must keep in mind that for the inverse hierarchy the seesaw mechanism implies a cancellation of the Dirac hierarchy for the third and second generation, and for the nearly degenerate spectrum also with the first generation, which seems unnatural, especially for the VO solution. Note that for zero mixing we have $m_{1}=m_{u}^{2} / M_{1}, m_{2}=m_{c}^{2} / M_{2}$ and $m_{3}=m_{t}^{2} / M_{3}$.

In this paper we follow a kind of inverse or bottom-up approach, namely we will determine $M_{R}$ from $M_{L}$ (and $M_{\nu}$ ). In the alternative direct or top-down approach both $M_{R}$ and $M_{\nu}$ are obtained from a theoretical framework and the inferred $M_{L}$ is matched to neutrino phenomenology. We would like to stress that for any precise choice of $M_{\nu}$ we can reproduce any form of $M_{L}$ by adjusting $M_{R}$. However, as we will see in the following sections, the form of both $M_{\nu}$ and $M_{R}$ has a crucial impact on the amount of baryon asymmetry generated in the leptogenesis mechanism, so that we can use the constraint from baryogenesis to study $M_{R}$ and $M_{\nu}$. Unfortunately, we are not yet able to determine the mass matrices with precision. Nevertheless, some important considerations, involving also the neutrinoless double beta decay, can be done and this would be a central issue of our paper.

\section{BARYOGENESIS FROM LEPTOGENESIS}

A baryon asymmetry can be generated from a lepton asymmetry [2]. In fact, this lepton asymmetry is produced by the out-of-equilibrium CP-violating decay of heavy neutrinos. The electroweak sphalerons [18], which violates $B+L$ but conserve $B-L$, transform part of this asymmetry into a baryon asymmetry. Then, the baryon asymmetry, 
defined as $Y_{B}=\left(n_{B}-n_{\bar{B}}\right) / 7 n_{\gamma}=\eta / 7$, where $n_{B}, n_{\bar{B}}, n_{\gamma}$ are number densities and $\eta$ is the baryon-to-photon ratio, can be written as (see Ref. [19,20] and references therein)

$$
Y_{B} \simeq \frac{1}{2} \frac{1}{g^{*}} d \epsilon_{1}
$$

with the $\mathrm{CP}$ violating asymmetry in the decay of the lightest heavy neutrino with mass $M_{1} \ll M_{2}<M_{3}$ given by

$$
\epsilon_{1} \simeq \frac{3}{16 \pi v^{2}}\left[\frac{\left[\left(M_{D}^{\dagger} M_{D}\right)_{12}\right]^{2}}{\left(M_{D}^{\dagger} M_{D}\right)_{11}} \frac{M_{1}}{M_{2}}+\frac{\left[\left(M_{D}^{\dagger} M_{D}\right)_{13}\right]^{2}}{\left(M_{D}^{\dagger} M_{D}\right)_{11}} \frac{M_{1}}{M_{3}}\right],
$$

where $M_{D}=M_{\nu} U_{R}$ and $U_{R}^{T} M_{R} U_{R}=D_{R}$, and $v \simeq m_{t}$ is the VEV of the Higgs doublet. The lightest heavy neutrino is in equilibrium during the decays of the two heavier ones, washing out the lepton asymmetry generated by them. The factor $1 / 2$ represents the part of the lepton asymmetry converted into a baryon asymmetry [21]. The parameter $g^{*} \simeq 100$ is the number of light degrees of freedom in the theory. Finally, the quantity $d$ is a dilution factor which mostly depends on the mass parameter

$$
\tilde{m}_{1}=\frac{\left(M_{D}^{\dagger} M_{D}\right)_{11}}{M_{1}}
$$

although for high values of $\tilde{m}_{1}$ some dependence on $M_{1}$ shows up. Minor dilution, $d$ of order $10^{-1}$, is obtained for $10^{-5} \mathrm{eV} \lesssim \tilde{m}_{1} \lesssim 10^{-2} \mathrm{eV}$, while outside this range the dilution grows (that is $d$ diminishes) 22, 23. In fact, if $\tilde{m}_{1}$ is too small, it is not possible to produce a sufficient number of heavy neutrinos at high temperature, while if $\tilde{m}_{1}$ is too large, the washout effect of lepton number violating scatterings is too strong and destroys the generated asymmetry. In order to be consistent with primordial nucleosynthesis, the baryon asymmetry $Y_{B}$ must be in the range $10^{-11}-10^{-10}$ 24]. At best, $Y_{B}$ is smaller than $\epsilon_{1}$ by three orders of magnitude. It is clear that when we obtain $M_{R}$ from $M_{\nu}$ and $M_{L}$ through the inverse seesaw formula (7), a determination of the baryon asymmetry is also achieved. In the following two sections we try a partial selection of mass matrices using the bound on the amount of baryon asymmetry.

\section{SYMPLIFIED ANALYSIS OF THE SEESAW MECHANISM}

In this section we determine the structure of the heavy neutrino mass matrix by assuming the diagonal form (5) for the Dirac neutrino mass matrix. In the next section the effect of a non diagonal form is discussed. In any subsection we write the zeroth order form of $M_{L}$, according to the three possible hierarchies of light neutrino masses, and then we study the heavy neutrino mass matrix and the implications for the baryon asymmetry and for the neutrinoless double beta decay. 


\section{A. Normal hierarchy}

For both kinds of mixings the zeroth order form for $M_{L}$ is given by

$$
M_{L} \sim\left(\begin{array}{ccc}
0 & 0 & 0 \\
0 & \frac{1}{2} & \frac{1}{2} \\
0 & \frac{1}{2} & \frac{1}{2}
\end{array}\right) m_{3},
$$

where a dominant block in the $\mu-\tau$ sector appears (see for example [25]). The overall scale is $m_{3} \sim 10^{-2}-10^{-1} \mathrm{eV}$.

\section{Double large mixing}

If there is full hierarchy of light neutrino masses, $m_{3} \gg m_{2} \gg m_{1}$, the matrix $M_{L}^{-1}$ takes a nearly democratic form [26], so that

$$
M_{R} \simeq\left(\begin{array}{ccc}
m_{u}^{2} & -m_{u} m_{c} & m_{u} m_{t} \\
-m_{u} m_{c} & m_{c}^{2} & -m_{c} m_{t} \\
m_{u} m_{t} & -m_{c} m_{t} & m_{t}^{2}
\end{array}\right) \frac{1}{m_{1}} .
$$

This matrix is diagonalized by the rotation

$$
U_{R} \simeq\left(\begin{array}{ccc}
1 & -\frac{m_{u}}{m_{c}} & \frac{m_{u}}{m_{t}} \\
\frac{m_{u}}{m_{c}} & 1 & -\frac{m_{c}}{m_{t}} \\
\frac{m_{u}}{m_{t}} & \frac{m_{c}}{m_{t}} & 1
\end{array}\right)
$$

with eigenvalues $M_{1} \simeq m_{u}^{2} / m_{1}, M_{2} \simeq m_{c}^{2} / m_{1}, M_{3} \simeq m_{t}^{2} / m_{1}$. For the full hierarchy and large solar mixing we have $m_{2}^{2} \sim 10^{-5} \mathrm{eV}^{2}$, hence $m_{1} \lesssim 10^{-3} \mathrm{eV}$ and the overall scale is $M_{R} \sim m_{t}^{2} / m_{1} \gtrsim 10^{16} \mathrm{GeV}$. In this section we take the full hierarchy as reference model.

If $m_{3} \gg m_{2} \simeq m_{1}$ (partially degenerate spectrum), we get

$$
M_{R} \simeq\left(\begin{array}{ccc}
m_{u}^{2} & -\epsilon m_{u} m_{c} & -\epsilon m_{u} m_{t} \\
-\epsilon m_{u} m_{c} & m_{c}^{2} & -m_{c} m_{t} \\
-\epsilon m_{u} m_{t} & -m_{c} m_{t} & m_{t}^{2}
\end{array}\right) \frac{1}{m_{1}}
$$


where very small elements $M_{R 12}$ and $M_{R 13}$ appear. The overall scale and the heavy masses do not change with respect to the full hierarchical case.

The further condition $m_{2}<0$ leads to the form

$$
M_{R} \simeq\left(\begin{array}{ccc}
\epsilon^{2} \frac{m_{1}}{m_{3}} m_{u}^{2} & -m_{u} m_{c} & m_{u} m_{t} \\
-m_{u} m_{c} & \left(\frac{m_{1}}{m_{3}}+\epsilon\right) m_{c}^{2} & \frac{m_{1}}{m_{3}} m_{c} m_{t} \\
m_{u} m_{t} & \frac{m_{1}}{m_{3}} m_{c} m_{t} & \left(\frac{m_{1}}{m_{3}}-\epsilon\right) m_{t}^{2}
\end{array}\right) \frac{1}{m_{1}} .
$$

If $m_{1} \gg \epsilon m_{3}$ the overall scale is lowered to $M_{R} \sim m_{t}^{2} / m_{3} \sim 10^{14} \mathrm{GeV}$. For $m_{1} \simeq \epsilon m_{3}$ we get the interesting form

$$
M_{R} \simeq\left(\begin{array}{ccc}
\epsilon^{3} m_{u}^{2} & -m_{u} m_{c} & m_{u} m_{t} \\
-m_{u} m_{c} & 2 \epsilon m_{c}^{2} & \epsilon m_{c} m_{t} \\
m_{u} m_{t} & \epsilon m_{c} m_{t} & 0
\end{array}\right) \frac{1}{m_{1}} .
$$

We stress the sharp difference between matrix (16) and matrices (12) or (14). While in (12) and (14) the largest element is $M_{R 33}$, in (16) it is $M_{R 13}$ or $M_{R 23}$. In the first case the structure of $M_{R}$ is roughly similar to the Dirac neutrino mass matrix $M_{\nu}$ in (5), that is a nearly diagonal form. In the second case, $M_{R}$ is roughly offdiagonal. As a consequence, also the overall mass scale is different, $m_{t}^{2} / m_{1} \gtrsim 10^{16} \mathrm{GeV}$ for the nearly diagonal form and $m_{u} m_{t} / m_{1} \gtrsim 10^{11} \mathrm{GeV}$ for the nearly offdiagonal form.

Let us discuss the implications for the baryogenesis via leptogenesis and for the neutrinoless double beta decay. The baryon asymmetry for the full normal hierarchy is $Y_{B} \sim 10^{-16}$. Due to the suppression of $M_{R 12}$ and $M_{R 13}$, the baryon asymmetry is smaller in the partially degenerate spectrum than in the full normal hierarchy. In fact, the relation $M_{D}^{\dagger} M_{D} \simeq M_{R} m_{1}$ holds in both cases, since $M_{D}^{\dagger} M_{D}$ and $M_{R} m_{1}$ are diagonalized by the same $U_{R}$ with the same eigenvalues. For the nearly offdiagonal form, the baryon asymmetry is enhanced to a sufficient level, due to the moderate hierarchy within $M_{R}$. In the neutrinoless double beta decay we get $M_{e e} \simeq m_{2} \sim 10^{-3}-10^{-2} \mathrm{eV}$ if $m_{2}>0$, and $M_{e e} \simeq\left(m_{2}^{2}-m_{1}^{2}\right) / m_{1,2} \sim 10^{-4}-10^{-3} \mathrm{eV}$ if $m_{2}<0$. Therefore, the nearly offdiagonal form for $M_{R}$ tends to enhance $Y_{B}$ but to suppress $M_{e e}$. Thus we have found that if the diagonal $M_{\nu}$ in (5) is assumed then in order to get sufficient baryon asymmetry the matrix $M_{R}$ must be roughly offdiagonal, which leads to a negative $m_{2}$ and an approximate prediction for $M_{e e}$, smaller by one order with respect to the case of a roughly diagonal $M_{R}$. The negative value for $m_{2}$ could be an indication that phases $\varphi_{1}$ and $\varphi_{2}$ are very different from each other. 


\section{Single large mixing}

In this case we have the form

$$
M_{R} \simeq\left(\begin{array}{ccc}
m_{u}^{2} & -\epsilon m_{u} m_{c} & -\epsilon m_{u} m_{t} \\
-\epsilon m_{u} m_{c} & \left(\frac{m_{1}}{m_{2}}+\epsilon^{2}\right) m_{c}^{2} & \left(\frac{m_{1}}{m_{2}}-\epsilon^{2}\right) m_{c} m_{t} \\
-\epsilon m_{u} m_{t} & \left(\frac{m_{1}}{m_{2}}-\epsilon^{2}\right) m_{c} m_{t} & \left(\frac{m_{1}}{m_{2}}+\epsilon^{2}\right) m_{t}^{2}
\end{array}\right) \frac{1}{m_{1}}
$$

and for $m_{1} \gg \epsilon^{2} m_{2}$ the structure of $M_{R}$ is nearly diagonal with the overall scale given by $m_{t}^{2} / m_{2} \sim 10^{15} \mathrm{GeV}$. The baryon asymmetry can get a moderate enhancement, because of the factor $m_{2} / m_{1}$, and $M_{e e} \simeq m_{1} \lesssim 10^{-4} \mathrm{eV}$. If $m_{1} \simeq \epsilon^{2} m_{2}$ the element $M_{R 23}$ vanishes. For $m_{2}<0$ and $m_{1} \simeq-\epsilon^{2} m_{2}$, we have a vanishing $M_{R 33}$, leading to a nearly offdiagonal form at a lower scale.

\section{B. Inverse hierarchy}

The zeroth order form of $M_{L}$ for spectrum $B_{1}$ is given by

$$
\begin{aligned}
M_{L} \sim\left(\begin{array}{ccc}
0 & -\frac{1}{\sqrt{2}} & \frac{1}{\sqrt{2}} \\
-\frac{1}{\sqrt{2}} & 0 & 0 \\
\frac{1}{\sqrt{2}} & 0 & 0
\end{array}\right) m_{1}, \\
M_{L} \sim\left(\begin{array}{ccc}
1 & 0 & 0 \\
0 & -\frac{1}{2} & \frac{1}{2} \\
0 & \frac{1}{2} & -\frac{1}{2}
\end{array}\right) m_{1},
\end{aligned}
$$

according to the double or single large mixing. For spectrum $B_{2}$ we have

$$
M_{L} \sim\left(\begin{array}{ccc}
1 & 0 & 0 \\
0 & \frac{1}{2} & -\frac{1}{2} \\
0 & -\frac{1}{2} & \frac{1}{2}
\end{array}\right) m_{1}
$$

for both cases. The overall scale is $m_{1,2} \sim 10^{-2}-10^{-1} \mathrm{eV}$. This is the same as the normal hierarchy, because both are determined by atmospheric oscillations. 


\section{Double large mixing}

The heavy neutrino mass matrix for spectrum $B_{2}$ is given by

$$
M_{R} \simeq\left(\begin{array}{ccc}
\frac{m_{3}}{m_{1}} m_{u}^{2} & \epsilon m_{u} m_{c} & \epsilon m_{u} m_{t} \\
\epsilon m_{u} m_{c} & m_{c}^{2} & m_{c} m_{t} \\
\epsilon m_{u} m_{t} & m_{c} m_{t} & m_{t}^{2}
\end{array}\right) \frac{1}{m_{3}} .
$$

If $m_{2}<0$, spectrum $B_{1}$, we get

$$
M_{R} \simeq\left(\begin{array}{ccc}
\epsilon^{2} m_{u}^{2} & -\frac{m_{3}}{m_{1}} m_{u} m_{c} & \frac{m_{3}}{m_{1}} m_{u} m_{t} \\
-\frac{m_{3}}{m_{1}} m_{u} m_{c} & m_{c}^{2} & m_{c} m_{t} \\
\frac{m_{3}}{m_{1}} m_{u} m_{t} & m_{c} m_{t} & m_{t}^{2}
\end{array}\right) \frac{1}{m_{3}} .
$$

In the inverse pattern there is stronger hierarchy in $M_{R}$ with respect to the full normal pattern, see the first row and column in (21) and (22). The form of $M_{R}$ is always nearly diagonal and the offdiagonal form cannot be realized. The mass scale is $M_{R} \sim m_{t}^{2} / m_{3} \gtrsim 10^{15}$ $\mathrm{GeV}$. Note also the difference between the inverse hierarchy and the partially degenerate spectrum in the element $M_{R 11}$, which is responsible for the inversion of the light neutrino masses $m_{1,2}$ and $m_{3}$.

As a consequence, the baryon asymmetry is even smaller than in the partially degenerate spectrum with $m_{2}>0$, because also $M_{1}$ is suppressed. Instead, the rate for neutrinoless double beta decay, related to $M_{e e}$, can be enhanced. In particular, for spectrum $B_{2}$ we have $M_{e e} \simeq m_{1,2} \sim 10^{-2}-10^{-1} \mathrm{eV}$, which is by one order higher than for the

full normal hierarchy. For spectrum $B_{1}$ we obtain $M_{e e} \simeq\left(m_{2}^{2}-m_{1}^{2}\right) / m_{1,2} \sim 10^{-4}-10^{-3}$ $\mathrm{eV}$.

\section{Single large mixing}

In this case the heavy neutrino mass matrix is the same as for the previous case with $m_{2}>0$, and $M_{e e} \simeq m_{1} \sim 10^{-2}-10^{-1} \mathrm{eV}$.

\section{Nearly degenerate spectrum}

Here the light masses are around $1 \mathrm{eV}$. The zeroth order form of $M_{L}$ for spectrum $C_{0}$ is diagonal, 


$$
M_{L} \sim\left(\begin{array}{lll}
1 & 0 & 0 \\
0 & 1 & 0 \\
0 & 0 & 1
\end{array}\right) m_{1},
$$

for both kinds of mixing. For spectrum $C_{1}$ we have

$$
\begin{gathered}
M_{L} \sim\left(\begin{array}{ccc}
0 & \frac{1}{\sqrt{2}} & -\frac{1}{\sqrt{2}} \\
\frac{1}{\sqrt{2}} & \frac{1}{2} & \frac{1}{2} \\
-\frac{1}{\sqrt{2}} & \frac{1}{2} & \frac{1}{2}
\end{array}\right) m_{1}, \\
M_{L} \sim\left(\begin{array}{ccc}
-1 & 0 & 0 \\
0 & 1 & 0 \\
0 & 0 & 1
\end{array}\right) m_{1},
\end{gathered}
$$

and for spectrum $C_{2}$

$$
\begin{gathered}
M_{L} \sim\left(\begin{array}{ccc}
0 & -\frac{1}{\sqrt{2}} & \frac{1}{\sqrt{2}} \\
-\frac{1}{\sqrt{2}} & \frac{1}{2} & \frac{1}{2} \\
\frac{1}{\sqrt{2}} & \frac{1}{2} & \frac{1}{2}
\end{array}\right) m_{1}, \\
M_{L} \sim\left(\begin{array}{lll}
1 & 0 & 0 \\
0 & 0 & 1 \\
0 & 1 & 0
\end{array}\right) m_{1},
\end{gathered}
$$

according to the double or single large mixing. Finally, for spectrum $C_{3}$

$$
M_{L} \sim\left(\begin{array}{ccc}
-1 & 0 & 0 \\
0 & 0 & 1 \\
0 & 1 & 0
\end{array}\right) m_{1}
$$

for both kinds of mixing. The overall scale is $m_{1,2,3} \sim 0.1-1 \mathrm{eV}$, determined by single beta decay. In the nearly degenerate spectrum several delicate cancellations among the terms of $M_{e e}$ may occur and our study of the heavy neutrino mass matrix is only indicative. 


\section{Double large mixing}

The heavy neutrino mass matrix for the spectra $C_{0}, C_{1}, C_{2}, C_{3}$ is respectively given by the forms

$$
\begin{gathered}
M_{R} \simeq\left(\begin{array}{ccc}
m_{u}^{2} & \rho m_{u} m_{c} & \rho m_{u} m_{t} \\
\rho m_{u} m_{c} & m_{c}^{2} & \epsilon^{2} m_{c} m_{t} \\
\rho m_{u} m_{t} & \epsilon^{2} m_{c} m_{t} & m_{t}^{2}
\end{array}\right) \frac{1}{m_{1}} \\
M_{R} \simeq\left(\begin{array}{ccc}
\epsilon^{2} m_{u}^{2} & m_{u} m_{c} & -m_{u} m_{t} \\
m_{u} m_{c} & m_{c}^{2} & m_{c} m_{t} \\
-m_{u} m_{t} & m_{c} m_{t} & m_{t}^{2}
\end{array}\right) \frac{1}{m_{1}}, \\
M_{R} \simeq\left(\begin{array}{ccc}
\epsilon^{2} m_{u}^{2} & -m_{u} m_{c} & m_{u} m_{t} \\
-m_{u} m_{c} & m_{c}^{2} & m_{c} m_{t} \\
m_{u} m_{t} & m_{c} m_{t} & m_{t}^{2}
\end{array}\right) \frac{1}{m_{1}}, \\
M_{R} \simeq\left(\begin{array}{ccc}
\epsilon m_{u} m_{c} & -\epsilon^{2} m_{c}^{2} & m_{c} m_{t} \\
\epsilon m_{u} m_{t} & m_{c} m_{t} & -\epsilon^{2} m_{t}^{2}
\end{array}\right) \frac{1}{m_{1}} .
\end{gathered}
$$

In (29), and (34) below, we write $\rho$ whenever a cancellation at the level of $\epsilon$ occurs. We see that matrices (29), (30), (31) are roughly close to the diagonal form, while (32) is not. The overall mass scale is $M_{R} \sim 10^{13} \mathrm{GeV}$.

For spectra $C_{0}$ and $C_{3}$ the baryon asymmetry is generally suppressed or much suppressed with respect to the full normal hierarchy, while for spectra $C_{1}$ and $C_{2}$ it is comparable. Instead, the rate for neutrinoless double beta decay can be further enhanced with respect to the inverse hierarchy. In particular, for spectra $C_{0}$ and $C_{3}$ we have $M_{e e} \simeq m_{1} \sim 0.1-1 \mathrm{eV}$, by one order higher than for the spectrum $B_{2}$. For spectra $C_{1}$ and $C_{2}$ we have cancellations leading to $M_{e e} \sim 10^{-5}-10^{-4} \mathrm{eV}$. 


\section{Single large mixing}

For spectra $C_{0}$ and $C_{3}$ we have the same matrices as for the previous mixing. For spectra $C_{1}$ and $C_{2}$ we get

$$
\begin{gathered}
M_{R} \simeq\left(\begin{array}{ccc}
-m_{u}^{2} & \epsilon m_{u} m_{c} & \epsilon m_{u} m_{t} \\
\epsilon m_{u} m_{c} & m_{c}^{2} & -\epsilon^{2} m_{c} m_{t} \\
\epsilon m_{u} m_{t} & -\epsilon^{2} m_{c} m_{t} & m_{t}^{2}
\end{array}\right) \frac{1}{m_{1}} \\
M_{R} \simeq\left(\begin{array}{ccc}
m_{u}^{2} & \rho m_{u} m_{c} & \rho m_{u} m_{t} \\
\rho m_{u} m_{c} & \epsilon^{2} m_{c}^{2} & m_{c} m_{t} \\
\rho m_{u} m_{t} & m_{c} m_{t} & \epsilon^{2} m_{t}^{2}
\end{array}\right) \frac{1}{m_{1}} .
\end{gathered}
$$

In this two cases the baryon asymmetry is suppressed or much suppressed with respect to the full normal hierarchy, and $M_{e e} \sim 0.1-1 \mathrm{eV}$.

\section{SEESAW MECHANISM AND BARYON ASYMMETRY}

In this section, instead of the diagonal form, we take the realistic mass matrices, expressed in terms of the Cabibbo parameter $\lambda=0.22$ and the overall mass scale,

$$
\begin{gathered}
M_{e} \sim\left(\begin{array}{ccc}
\lambda^{6} & \lambda^{3} & \lambda^{5} \\
\lambda^{3} & \lambda^{2} & \lambda^{2} \\
\lambda^{5} & \lambda^{2} & 1
\end{array}\right) m_{b}, \\
M_{\nu} \sim\left(\begin{array}{lll}
\lambda^{12} & \lambda^{6} & \lambda^{10} \\
\lambda^{6} & \lambda^{4} & \lambda^{4} \\
\lambda^{10} & \lambda^{4} & 1
\end{array}\right) m_{t} .
\end{gathered}
$$

These forms can be motivated by an $U(2)$ horizontal symmetry, see Ref. 27] and references therein. Again we neglect $U_{e}$ with respect to $U$. However, the effect of $U_{\nu} \neq \mathbb{1}$ is crucial. Of course, $M_{L}^{-1}$ can be obtained from the previous section by deleting the quark masses 
in $M_{R}$. In the following calculation we will assume that no cancellations occur between two quantities of the same order in $\lambda$.

For the full normal hierarchy and double large mixing we get

$$
M_{R} \sim\left(\begin{array}{ccc}
\lambda^{12} & \lambda^{10} & \lambda^{6} \\
\lambda^{10} & \lambda^{8} & \lambda^{4} \\
\lambda^{6} & \lambda^{4} & 1
\end{array}\right) \frac{m_{t}^{2}}{m_{1}},
$$

diagonalized by

$$
U_{R} \sim\left(\begin{array}{ccc}
1 & \lambda^{2} & \lambda^{6} \\
-\lambda^{2} & 1 & \lambda^{4} \\
\lambda^{6} & -\lambda^{4} & 1
\end{array}\right),
$$

with eigenvalues $M_{3} \sim m_{t}^{2} / m_{1}, M_{2} \sim \lambda^{8} M_{3}, M_{1} \sim \lambda^{12} M_{3}$, and consistent with the $U(2)$ horizontal symmetry [27]. The baryon asymmetry is enhanced with respect to the diagonal case but remains too small, $Y_{B} \sim 10^{-14}$. In fact, the relation $M_{D}^{\dagger} M_{D} \sim M_{R} m_{1}$ holds, and one obtains

$$
\epsilon_{1} \sim \frac{3}{16 \pi}\left(\frac{\lambda^{20}}{\lambda^{12}} \lambda^{4}+\frac{\lambda^{12}}{\lambda^{12}} \lambda^{12}\right) \sim 10^{-10},
$$

and $\tilde{m}_{1} \sim m_{1}$. The same $M_{R}$ and $Y_{B}$ come out for the partially degenerate spectrum with $m_{2}>0$, the inverse hierarchy, and the nearly degenerate spectra $C_{1}$ and $C_{2}$, although the scale of $M_{R}$ is changed accordingly.

For the partially degenerate spectrum with $m_{2}<0$, assuming for example both $\epsilon \sim \lambda^{4}$ and $M_{R 33} \sim \lambda^{12} m_{t}^{2} / m_{1}$, we obtain

$$
M_{R} \sim\left(\begin{array}{ccc}
\lambda^{16} & \lambda^{12} & \lambda^{10} \\
\lambda^{12} & \lambda^{10} & \lambda^{6} \\
\lambda^{10} & \lambda^{6} & \lambda^{8}
\end{array}\right) \frac{m_{t}^{2}}{m_{1}}
$$

By considering $M_{R}^{\dagger} M_{R}$, one finds that $M_{R}$ is diagonalized by

$$
U_{R} \sim\left(\begin{array}{ccc}
1 & \lambda^{4} & \lambda^{6} \\
-\lambda^{4} & 1 & \lambda^{2} \\
\lambda^{6} & -\lambda^{2} & 1
\end{array}\right),
$$


with eigenvalues $M_{3} \sim \lambda^{6} m_{t}^{2} / m_{1}, M_{2} \sim M_{3}, M_{1} \sim \lambda^{4} M_{3}$. Then we obtain

$$
\epsilon_{1} \sim \frac{3}{16 \pi}\left(\frac{\lambda^{16}}{\lambda^{12}} \lambda^{4}+\frac{\lambda^{12}}{\lambda^{12}} \lambda^{4}\right) \sim 10^{-4},
$$

and $\tilde{m}_{1} \sim \lambda^{2} m_{1}$, so that a sufficient amount of baryon asymmetry can be easily achieved. Note that here the dominant term in the leptogenesis formula is the second one. The mass scale in (37) is given by $m_{t}^{2} / m_{1} \gtrsim 10^{16} \mathrm{GeV}$, and in (40) by $\lambda^{6} m_{t}^{2} / m_{1} \gtrsim 10^{12} \mathrm{GeV}$.

For spectrum $C_{0}$ we have the nearly diagonal form

$$
M_{R} \sim\left(\begin{array}{ccc}
\lambda^{12} & \lambda^{10} & \lambda^{10} \\
\lambda^{10} & \lambda^{8} & \lambda^{4} \\
\lambda^{10} & \lambda^{4} & 1
\end{array}\right) \frac{m_{t}^{2}}{m_{1}}
$$

and for spectrum $C_{3}$

$$
M_{R} \sim\left(\begin{array}{ccc}
\lambda^{16} & \lambda^{10} & \lambda^{6} \\
\lambda^{10} & \lambda^{8} & \lambda^{4} \\
\lambda^{6} & \lambda^{4} & \lambda^{4}
\end{array}\right) \frac{m_{t}^{2}}{m_{1}}
$$

The baryon asymmetry is very small in the case $C_{0}$ and moderate in the case $C_{3}$.

For the single large mixing the results are roughly similar to the double large mixing. Few changes can be easily shown and are not discussed here. Thus we see that using non diagonal Dirac neutrino mass matrices and doing an order-of-magnitude analysis lead to few forms for the heavy neutrino mass matrix. Then the two questions of determining the symmetry generating mass matrices and discovering their coefficient should be addressed. For example, horizontal $U(1)$ or $U(2)$ symmetries can be used. This is a fundamental subject that we will try to discuss elsewhere. We need not start from matrices (35), (36) but other forms are possible as well [28].

A simple way to enhance the baryon asymmetry is by means of a quite moderate hierarchy in the Dirac neutrino mass matrix, that is for its eigenvalues and/or for its mixing angles [29,30]. This is quite evident from the leptogenesis formula. For example, instead of (36), one can adopt

$$
M_{\nu} \sim\left(\begin{array}{ccc}
\lambda^{6} & \lambda^{3} & \lambda^{5} \\
\lambda^{3} & \lambda^{2} & \lambda^{2} \\
\lambda^{5} & \lambda^{2} & 1
\end{array}\right) m_{t}
$$


that is a matrix similar to charged lepton masses but with the same overall scale as up quark masses. Let us discuss this issue. In section III we have assumed $M_{e} \sim M_{d}$ and $M_{\nu} \sim M_{u}$. This is the simplest hypothesis within the standard model, and in the supersymmetric model can be motivated by the fact that the two pairs $M_{e, d}$ and $M_{\nu, u}$ are generated by two distinct Higgs doublets. However, Yukawa couplings for the Dirac neutrino can be very different from the Yukawa couplings for the up quarks. If this case occurs, the hierarchy of masses and mixings in the Dirac neutrino sector can be very different from the mass hierarchy of up quarks and the CKM quark mixing, respectively. When we take matrices (35) and (45) we obtain

$$
\begin{gathered}
M_{R} \sim\left(\begin{array}{ccc}
\lambda^{6} & \lambda^{5} & \lambda^{3} \\
\lambda^{5} & \lambda^{4} & \lambda^{2} \\
\lambda^{3} & \lambda^{2} & 1
\end{array}\right) \frac{m_{t}^{2}}{m_{1}}, \\
\epsilon_{1} \sim \frac{3}{16 \pi}\left(\frac{\lambda^{10}}{\lambda^{6}} \lambda^{2}+\frac{\lambda^{6}}{\lambda^{6}} \lambda^{6}\right) \sim 10^{-6},
\end{gathered}
$$

and a sufficient amount of baryon asymmetry, $Y_{B} \sim 10^{-10}$. Both the internal hierarchy and the overall scale of $M_{\nu}$ are important. In fact, if the overall scale in (45) is $m_{b, \tau}$ instead of $m_{t}$, the baryon asymmetry again suppressed [29]. The relation $M_{\nu} \sim(\tan \beta) M_{e}$ can be obtained within supersymmetric left-right models [31. For very large $\tan \beta$ we get a Dirac neutrino mass matrix similar to (45).

\section{DISCUSSION}

Assuming a nearly diagonal mass matrix for Dirac neutrinos, we have studied the structure of the Majorana mass matrix of right-handed neutrinos within the seesaw framework, and its implications for the baryogenesis via leptogenesis and for the neutrinoless double beta decay. Then we have explored the effect of a non diagonal mass matrix for Dirac neutrinos. In this context, we find few possibilities to obtain a sufficient level of baryon asymmetry. The only case when the asymmetry is large should be the nearly offdiagonal form of $M_{R}$. Usually, the behaviour of $M_{e e}$ is opposite to $Y_{B}$, namely when $Y_{B}$ is suppressed $M_{e e}$ is enhanced and viceversa. For the offdiagonal form we get $M_{e e} \sim 10^{-4}-10^{-3} \mathrm{eV}$. In the supersymmetric formula for the leptogenesis, the baryon asymmetry is only slightly enhanced [19,22. In fact, although there are new decay channels, also the washout process is stronger. 
When a moderate hierarchy in $M_{\nu}$ is adopted, as in (45), then $M_{R}$ need not be close to the offdiagonal form. Therefore, instead of $M_{e e} \sim 10^{-4}-10^{-3} \mathrm{eV}$, we can yield the value $M_{e e} \sim 10^{-3}-10^{-2} \mathrm{eV}$ for the normal hierarchy and $M_{e e} \sim 10^{-2}-10^{-1} \mathrm{eV}$ for the inverse hierarchy. Note that these predictions cover three different ranges of values for $M_{e e}$, so that informations from the neutrinoless double beta decay could clarify the structure of fermion mass matrices, if the leptogenesis mechanism is valid.

As a conclusion, we find that, if $Y_{B}$ has to be within the allowed range, then retaining quark-lepton mass relations $M_{e} \sim M_{d}$ and $M_{\nu} \sim M_{u}$ leads to the roughly offdiagonal form for $M_{R}$ and the prediction $M_{e e} \sim 10^{-4}-10^{-3} \mathrm{eV}$. If $M_{\nu} \sim M_{u}$ is not true, then $M_{R}$ can be roughly close to the diagonal form and $M_{e e}$ larger than $10^{-3} \mathrm{eV}$, with $Y_{B}$ in the allowed range. This is the central result of our paper. We have proposed such two different kinds of model in the previous section.

It has been suggested [26] that the nearly offdiagonal form for $M_{R}$ is consistent with the nonsupersymmetric unified model $S O(10)$ with an intermediate symmetry breaking scale, where the heavy neutrino mass is generated, while the nearly diagonal form for $M_{R}$ is consistent with the supersymmetric version without such an intermediate scale, the heavy neutrino mass being generated at the unification scale. In both cases, quark-lepton symmetry is valid. Then, we ask if the framework described here could be embedded in unified models. On the one hand, the leptogenesis scenario could work within unified models [32]. On the other hand, unified model have not yet received decisive support from the experimental detection of proton decay, so that we find attractive to keep the minimal scenario, as was the motivation of the original paper on the leptogenesis [2]. Within a unified framework, the leptogenesis constraint favours the nonsupersymmetric model.

The present paper focuses on the general structure of mass matrices and does not exclude that possible fine tuning could produce a sufficient amount of baryon asymmetry [19,33], even with nearly diagonal mass matrices. Other interesting papers on the relation between leptogenesis and mass matrices are reported in Ref. [34].

In our simple approach we have not considered the effect of running masses and mixings from the low scale to the high $M_{R}$ scale where the seesaw formula (6) applies. At our level of approximation, only for the supersymmetric version with large $\tan \beta$ they can be significant [35]. In particular, for spectrum $C_{0}$ both the double and the single large mixing are converted to nearly zero mixing, and for spectra $B_{2}$ and $C_{3}$ the double large mixing is converted to single large mixing. Such spectra are characterized by degeneracy in mass and sign. However, it has been argued [36] that the running of vacuum expectation values could improve the stability of the lepton mixing matrix. 
[1] M. Gell-Mann, P. Ramond and R. Slansky, in Supergravity, eds. P. van Nieuwenhuizen and D. Freedman (North Holland, Amsterdam, 1979)

T.Yanagida, in Proceedings of the Workshop on Unified Theories and Baryon Number in the Universe, eds. O. Sawada and A. Sugamoto (KEK, Tsukuba, 1979)

R.N. Mohapatra and G. Senjanovic, Phys. Rev. Lett. 44 (1980) 912

[2] M. Fukugita and T. Yanagida, Phys. Lett. B 174 (1986) 45

[3] P. Langacker, R.D. Peccei and T. Yanagida, Mod. Phys. Lett. A 1 (1986) 541

M.A. Luty, Phys. Rev. D 45 (1992) 455

C.E. Vayonakis, Phys. Lett. B 286 (1992) 92

R.N. Mohapatra and X. Zhang, Phys. Rev. D 46 (1992) 5331

W. Buchmuller and M. Plumacher, Phys. Lett. B 389 (1996) 73

R. Rangarajan and H. Mishra, Phys. Rev. D 61 (2000) 043509

[4] S. Weinberg, Phys. Rev. Lett. 43 (1979) 1566

[5] Z. Maki, M. Nakagawa and S. Sakata, Prog. Theor. Phys. 28 (1962) 870

S.M. Bilenky and B. Pontecorvo, Phys. Lett. B 95 (1980) 233

[6] N. Cabibbo, Phys. Rev. Lett. 10 (1963) 531

M. Kobayashi and T. Maskawa, Prog. Theor. Phys. 49 (1973) 652

L.L. Chau and W.Y. Keung, Phys. Rev. Lett. 53 (1984) 1802

[7] G.L. Fogli, E. Lisi, D. Montanino, A. Palazzo, Phys. Rev. D 64 (2001) 093007

J.N. Bahcall, M.C. Gonzalez-Garcia, C. Pena-Garay, J. High Energy Phys. 08 (2001) 014

A. Baudyopadhyay, S. Choubey, S. Goswami, K. Kar, Phys. Lett. B 519 (2001) 83

P.I. Krastev and A.Yu. Smirnov, Phys. Rev. D 65 (2002) 073022

Q.R. Ahmad et al., nucl-ex/0204009

[8] E.Kh. Akhmedov, Phys. Lett. B 467 (1999) 95

[9] M. Apollonio et al., Phys. Lett. B 420 (1998) 397; 466 (1999) 415. See also the Palo Verde experiment: F. Boehm et al., Phys. Rev. D 62 (2000) 072002

[10] H.V. Klapdor-Kleingrothaus et al., Eur. Phys. J. A 12 (2001) 147

[11] F. Feruglio, A. Strumia and F. Vissani, hep-ph/0201291

[12] H.V. Klapdor-Kleingrothaus et al., Mod. Phys. Lett. A 16 (2001) 2409

[13] C.E. Aalseth et al., hep-ex/0202018

[14] W. Rodejohann, Phys. Rev. D 62 (2000) 013011

[15] G. Altarelli and F. Feruglio, Phys. Rep. 320 (1999) 295 
[16] J. Bonn et al., Nucl. Phys. B (Proc. Suppl.) 91 (2001) 273

V. Lobashev et al., Nucl. Phys. B (Proc. Suppl.) 91 (2001) 280

[17] D. Falcone, hep-ph/0105124

[18] V.A. Kuzmin, V.A. Rubakov and M.E. Shaposhnikov, Phys. Lett. B 155 (1985) 36

[19] D. Falcone and F. Tramontano, Phys. Rev. D 63 (2001) 073007; Phys. Lett. B 506 (2001) 1

F. Buccella, D. Falcone and F. Tramontano, Phys. Lett. B 524 (2002) 241

[20] L. Covi, E. Roulet and F. Vissani, Phys. Lett. B 384 (1996) 169

[21] J.A. Harvey and M.S. Turner, Phys. Rev. D 42 (1990) 3344

S.V. Khlebnikov and S.E. Shaposhnikov, Nucl. Phys. B 308 (1988) 885

[22] W. Buchmuller and M. Plumacher, Int. J. Mod. Phys. A 15 (2000) 5047

[23] M. Hirsch and S.F. King, Phys. Rev. D 64 (2001) 113005

[24] K.A. Olive, hep-ph/0202486

[25] W. Buchmuller and T. Yanagida, Phys. Lett. B 445 (1999) 399

[26] D. Falcone, Phys. Lett. B 479 (2000) 1; hep-ph/0006130

[27] D. Falcone, Phys. Rev. D 64 (2001) 117302

[28] B.R. Desai and A.R. Vaucher, Phys. Rev. D 63 (2001) 113001

J.L. Chkareuli and C.D. Froggatt, Phys. Lett. B 450 (1999) 158

[29] D. Falcone, Phys. Rev. D 65 (2002) 077301

[30] M.S. Berger and K. Siyeon, Phys. Rev. D 65 (2002) 053019

[31] K.S. Babu, B. Dutta and R.N. Mohapatra, Phys. Rev D 60 (1999) 095004; Phys. Lett. B 458 (1999) 93

[32] A. Pilaftsis, Int. J. Mod. Phys. A 14 (1999) 1811

S. Carlier, J.M. Frere and F.S. Ling, Phys. Rev D 60 (1999) 096003

E. Ma, S. Sarkar and U. Sarkar, Phys. Lett. B 458 (1999) 73

[33] G.C. Branco, R. Gonzalez Felipe, F.R. Joaquim and M.N. Rebelo, hep-ph/0202030

E. Nezri and J. Orloff, hep-ph/0004227

[34] J. Ellis, S. Lola and D. Nanopoulos, Phys. Lett. B 452 (1999) 87

M.S. Berger, Phys. Rev. D 62 (2000) 013007

R. Barbieri, P. Creminelli, A. Strumia, N. Tetradis, Nucl. Phys. B 575 (2000) 61

W. Buchmuller and D. Wyler, Phys. Lett. B 251 (2001) 291 
[35] N. Haba and N. Okamura, Eur. Phys. J. C 14 (2000) 347

N. Haba, Y. Matsui and N. Okamura, Eur. Phys. J. C 17 (2000) 513

[36] N. Nimai Singh, Eur. Phys. J. C 19 (2001) 137; hep-ph/0112123 